\title{
Phenotypic Assays in Drug Discovery: a resurgence
}

\author{
Ram Ramabhadran* \\ Independent Consultant, USA \\ *Corresponding author: Ram Ramabhadran Independent Consultant, 204 Running Creek Rd, Cary NC 27518, USA
}

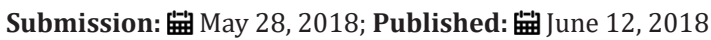

Abbreviations: PDD: Phenotypic Drug Discovery; HTS: High Throughput Screening; GPCRs: G-protein coupled receptors; TDD: Target based Drug Discovery; NCATS: National Center for Advancing Translational Sciences; NIH: National Institutes of Health; DARPA: Defense Advanced Research Project Agency ; FDA: Food and Drug Administration; iPSCs: Induced Pluripotent Stem Cells; OOC: Organ-on-a-chip

\section{Introduction}

Prior to 1980s drug discovery in the pharmaceutical industry relied on the use of whole animals or organ explants. Typically, compounds or classes of compounds synthesized by chemists specifically for pharmaceutical applications (or for another such as the dye industry) were tested in an array of assays that relied on measuring phenotypic changes (morphological, physiological, electrophysiological, etc.) in animals, organ explants, or tissue extracts [1]. This approach yielded a cohort of powerful drugs in use even today [2]. These leading discoveries have in turn served as a springboard superior follow on drugs based on chemical modifications of the original "First-in-class" molecules. In other words drug discovery in this era was based solely on Phenotypic Drug Discovery (PDD) approaches.

The approach to drug discovery acquired a new facet since the advent of recombinant DNA technology in the 1980's, [3,4]. Advances in molecular and cell biology have contributed to understanding of major biological pathways, biochemical/physiological/genetic mechanisms of disease causation and identification of "drug gable" targets. Ever since, a substantial proportion of small molecule drug discovery has relied predominantly on high throughput screening (HTS) against specific recombinant target proteins such as purified enzymes (e.g., kinases), and G-protein-coupled receptors (GPCRs) and ion channels, expressed in artificial recombinant cells systems, resulting in many novel drugs in every sector of medicine. This approach, in contrast to PDD that it superseded, has been dubbed Target-based Drug Discovery (TDD) [4,5].

Both TDD and PDD have their respective strengths and weaknesses [4]. TDD is a reductionist approach that relies on modulating a specific target protein aided by complete structural and functional information and a mechanistic understanding of its role in a biochemical pathway and in disease, This information is often deciphered using biochemical or biophysical methods or gene knock-out and/or gene over expression in transgenic animals.

Knowledge target characteristics facilitates the creation of efficient engineered screening systems that can be interrogated using high throughput methods permitting the screening of large numbers of compounds. Structural information about the target enables further refinement of hits discovered by TDD through molecular biological, biochemical, structural and in silico approaches; Hence TDD generates the sense of a 'rational' approach that is alluring to scientists in contrast to the 'blind' screening engendered by PDD. TDD's weakness lies in its rather narrow focus on a single target in an engineered system removed from the cellular situation relevant to the complex disease state. Because of the incomplete understanding of these highly complex of biological networks, TDD can result in the putative drug's failure to treat the disease despite its high affinity for its target. In fact, an examination of the previously approved drugs discovered through PDD approaches indicates that many of them exhibit 'poly pharmacology', i.e., interaction with multiple targets driving both their efficacy and side effects[6]; It is estimated that on average, current PDD-based drugs, it is interact with six different targets[7]. Therefore, as has been with many approved TDD-based drugs, it is very likely that drugs produced by TDD interact to various degrees with family members of the primary target as well as with unknown targets $[7,8]$.

Conversely, PDD depends on phenotypic changes as its readout without detailed knowledge of the disease mechanisms or targets, and hence is target agnostic. In contrast to TDD's focus on a single target screening in the PDD mode interrogates the system as a whole and thus can potentially uncover compounds act on multiple relevant disease targets .This can result in the identification of compounds acting via different mechanisms through poly 
pharmacology. However, insufficient knowledge of the target(s) and the candidate compound's molecular mechanisms of action makes refinement of the hits harder than in the TDD approach. Further, PDD has significantly lower throughput relative to TDD. It is noteworthy however, that even with the dominance of TDD approaches in today's field, many PDD assays were/are used for secondary validation of TDD hits [4-9].

The recombinant DNA revolution and the subsequent advent and high expectation placed on TDD soon overtook the use of PDD in the Pharma industry. Many authors have since attributed the industry's lack of productivity to the over-reliance on TDD at the expense of PDD [4]. Nonetheless, an analysis of new molecular entities(NMEs) among drugs approved by the FDA between 1999 and 2008 shows that among a 45 first-in-class drugs, a significantly higher proportion have been discovered using PDD (37\% vs $23 \%$ via TDD)[4]. Although these exact figures on the relative productivity of PDD vs. TDD are debated, PDD's continued relevance as a vital component of drug discovery(despite TDD's allure) has drawn increasing attention over the years [7].

Along with the conspicuous increase in the industry's use of PDD major advances in molecular and cellular biology have significantly enhanced PDD's prospects [7]. Further, concurrent developments in instrumentation such as advanced microscopic techniques and computerized image analysis methods [10] have given rise powerful tools for the design and execution of phenotypic assays. These leaps in technology have enabled novel assays ranging from cell-based assays to assays based on whole animals. On the cellular front, advances in tissue culture technologies have permitted the creation of novel screens using primary cells, derived from patients in some cases, by often seeding them in appropriate 3-dimensional matrices that to various degrees, simulates their natural environment [11,12]. Novel devices dubbed 'organ on chip'(OOC) contain cells from the desired organ seeded on to the "chip" on appropriate substrates with necessary modifications and perfusion of the 'chip' to mimic the in vivo environment in the organ [13].

In other cases, cellular organoids have been developed [14] A prime example \& an example of the growth in cell-based PDD screens is, the highly coordinated national level effort initiated in the US towards the development of human tissue chips. This effort includes the National Center for Advancing Translational Sciences (NCATS) of the National Institutes of Health (NIH), the Defense Advanced Research Project Agency (DARPA) and the Food and Drug Administration (FDA); The partnership long-range goal is to develop tissue chips for drug discovery and safety testing to be validated in academic and industrial labs; similar efforts are in progress in Europe and Japan [13]. Such collaborations among government, industry and academia bode well for the acceleration and adoption of OOC based PDD for drug discovery.

Alongside Two stellar developments have opened new avenues for PDD, namely the discovery of pluripotent stem cells and the methods for artificially inducing pluripotency into non-stem cells such as fibroblasts resulting in induced pluripotent cells (iPSCs), Stem cells derived from healthy volunteers and patients can be differentiated efficiently into those of the organs involved in diseases, providing ideal screening systems for PDD; for instance, fibroblasts derived from patient skin biopsies can be induced to become pluripotent and then differentiated into neurons or cardiomyocytes, etc. This approach has been leveraged to design assays for indication areas such as cardiology, neurology, and for toxicology [15-17].

Extensive efforts using cell-based assays are now devoted to the discovery of new drugs as well as repurposing of both existing efficacious drugs, and drug candidates that failed to show efficacy in late clinical trials. Such repurposing provides an expedited path to new drugs as most of these compounds can move directly to the clinic having already undergone human safety study requirements typical of totally new molecular entities. This is the rationale behind the launch of, an NCATS-industry partnership (featuring several major pharmaceutical companies) titled "Discovering New Therapeutic Uses for Existing Molecules” (https://ncats.nih.gov/ ntu) where PDD plays a significant role [18].

Moving to a higher level of organization and complexity, a variety of whole animals, generally known as 'model organisms' has also been employed in PDD. Model organisms ranging from bacteria through worms and fish to rodents have been used in novel ways as phenotypic screens [11-19]. The relevance and the appropriateness of an organism depend on the phylogenetic distance of the model organism from humans, and the nature of applications. Accordingly while bacteria, fungi and worms are clearly necessary choices for anti-infective PDD, in for metabolic diseases such as cancer and diabetes rodents are far more appropriate given their genetic similarity to humans. Based on this rationale, although academic laboratories have used lower organisms such as yeasts and worms for developing screening assays for some diseases, the pharmaceutical industry has relied exclusively on vertebrates, notably mice, This trend is further facilitated by the abundance of transgenic and knock-out mouse lines, developed as disease models. Clearly the use of more human relevant organism is associated with an increase in cost and a decrease in throughput. Nonetheless, yeast, worms, flies, fish and other lower organisims are ideal for delineating gene function where homologs of human genes occur, and thus facilitate target identification for TDD [19].

Due to the availability of mouse models for a wide range of diseases, multiplexed screening platforms using mice have been developed despite the lower throughput and higher labor intensiveness. One such multiplex platform combining approximately 40 disease models across 14 broad therapeutic indication areas has been successfully applied to drug repurposing efforts [20]. Another repurposing effort utilizes a suite of automated behavioral assays that rely on computer vision to extract multiple ( 2000) behavioral features from drug-treated mice generating a behavioral profile of a large set of known psychotropic drugs and drug candidate compounds. This information can be analyzed using bio informatics techniques to produce behavioral spectra of 
the prototype drugs and candidates, enabling drug repurposing through meaningful comparisons [21], the inclusion of disease models can further expand the power of this approach permitting the interrogating compounds for the reversal of disease phenotypes.

\section{Conclusion}

Though TDD has displaced PDD from drug discovery's center stage since the 1980s, recent analyses have confirmed the power and utility of PDD to discover drugs on par with TDD. Given the renewed appreciation and enthusiasm within the drug discovery community PDD's value within in the drug discovery community and the added impact of advances in cell culture, animal models and automated instrumentation, of PDD's future as a driver of drug discovery appears bright.

\section{Acknowledgements}

I am grateful to Dr. Richard Wiggins and Sanjeev Ramabhadran for their valuable suggestions and corrections.

\section{References}

1. Vogel HG (2008) Introduction. In Drug Discovery and Evaluation Pharmacological Assays, ed. HG Vogel New York Springer Verlag: 1-45.

2. Wikipedia List of drugs by year of discovery. https://en.wikipedia.org/ wiki/List_of_drugs_by_year_of_discovery 2018.

3. Dollery C T (1999) Drug discovery and development in the molecular era. J Clin Pharmacol 47: 5-6.

4. Swinney DC, J Anthony (2011) Analysis How were new medicines discovered? Nature Reviews Drug Discovery 10: 507-519.

5. Moffat JG et al, (2017) Opinion : Opportunities and challenges in phenotypic drug discovery: an industry perspective. Nature Reviews Drug Discovery 16: 531-543

6. Hopkins AL (2012) A case for poly pharmacology, in Polypharmacology in Drug Discovery. P J-U Peters Editor John Wiley and Sons: Hoboken New Jersey p. 1-6.

7. Haasen D et al, (2017) How Phenotypic Screening Influenced Drug Discovery: Lessons from Five Years of Practice. Assay and Drug Development Technologies 15: 239-246.
8. Car BD (2012) The relevance of off target poly pharmacology in Polypharmacology In Drug Discovery .JU Peters Editor Hoboken New Jersey 9-14.

9. Lee JA, EL Berg (2013) Neoclassic drug discovery the case for lead generation using phenotypic and functional approaches. Journal of bio molecular screening. 18: 1143-1155.

10. Boutros M F, Heigwer, C Laufer (2015) Microscopy Based High Content Screening. Cell 163: 1314-1325.

11. Szabo M et al, (2017) Cell and small animal models for phenotypic drug discovery. Drug Design Development and Therapy 11: 1957-1967.

12. Edmondson R et al, (2014) Three-dimensional cell culture systems and their applications in drug discovery and cell-based biosensors. Assay and Drug Development Technologies 12(4): 207-218.

13. Ronaldson Bouchard K, G Vunjak Novakovic (2018) Organs on a Chip: A Fast Track for Engineered Human Tissues in Drug Development. Cell Stem Cell 22: 310-324.

14. Benbrook DM (2006) Organotypic cultures represent tumor microenvironment for drug testing. Drug Discovery Today Disease Models 2: 143-148.

15. Avior Y I Sagi, N Benvenisty (2016) Pluripotent stem cells in disease modelling and drug discovery. Nature Reviews Molecular Cell biology 17: $170-182$.

16. Ursu A, HR Scholer, H Waldmann (2017) Small molecule phenotypic screening with stem cells. Nature Chemical Biology 13: 560-563.

17. Wagner BK, SL Schreiber (2016) The Power of Sophisticated Phenotypic Screening and Modern Mechanism of Action Methods. Cell Chemical biology 23: 3-9.

18. Lee JA, et al (2015) Novel Phenotypic Outcomes Identified for a Public Collection of Approved Drugs from a Publicly Accessible Panel of Assays. PLOS ONE 10: e0130796.

19. Strange K (2016) Drug Discovery in Fish Flies and Worms. ILAR Journal 57: 133-143.

20. Ciallella JR, AG Reaume (2017) In vivo phenotypic screening: clinical proof of concept for a drug repositioning approach. Drug Discovery Today Technologies 23: 45-52.

21. Brunner D, et.al (2012) Behavior Based Screening as an Approach to Poly pharmacological Ligands In Polypharmacology in Drug Discovery. JU Peters Editor Wiley and Sons Hoboken New Jersey pp. 303-313.
Creative Commons Attribution 4.0 International License

For possible submissions Click Here
Submit Article

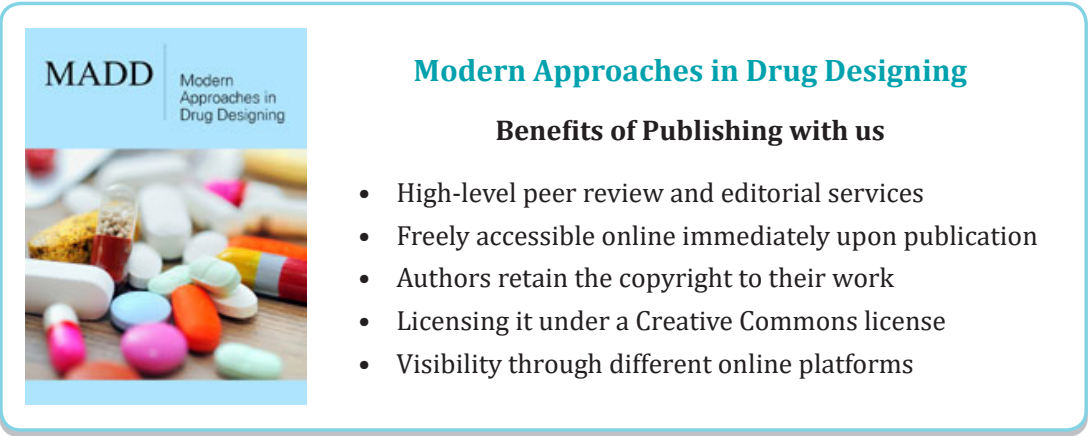

